\title{
ENTREPRENEURIAL INTENTION AMONG STUDENTS OF ARTS AND SCIENCE COLLEGES IN CHENNAI - AN EMPIRICAL STUDY
}

\author{
P.Jayalakshmi1, Dr.A.S.Saranya² \\ ${ }^{1}$ Research Scholar Commerce Department, Ethiraj College for Women \\ ${ }^{2}$ Commerce Department, Ethiraj College for Women \\ Email:pjlakshmipandian@yahoo.co.in
}

\section{Abstract-}

Entrepreneurs play a major role in accelerating the pace of economic development with their innovative and creative approach to produce goods and services. This article examines whether students' perception on 'risk taking propensity', 'internal locus of control', attitude towards entrepreneurship, perceived barriers, perceived support and 'entrepreneurial intent' vary based on the demographic variables. The analysis was done using student t-test and Anova. The data for this research paper was collected from students pursuing their final year in arts and science colleges in Chennai. The results of the research paper reveal that the Risk Taking Propensity and Attitude towards Entrepreneurship contribute significantly towards Entrepreneurial Intent. The research findings have implications for educators and policy makers.

Keywords: Entrepreneurial Intention; Risk Taking Propensity, Internal Locus of Control, Perceived Barriers; Perceived Support.

\section{BRIEF BACKGROUND OF THE STUDY}

Entrepreneurs are the pillars on which the economic health of societies is built. Entrepreneurship has been identified as the fourth factor of production. Entrepreneurship is very important for further social development through increased job opportunities and consequent economic prosperity. The term 'entrepreneur' was first coined by the French economist Richard Cantillon in the seventeenth century (Cantillon, 1755).He defined entrepreneur as 'an agent who buys means of production at certain prices in order to combine them into a product that he is going to sell at prices that are uncertain at the moment at which he commits himself to cost'.

Say (1821) defined entrepreneur as 'an economic agent who unites all means of production-the labour of one, the capital or land of the other and who finds the value of products which result from their employment, reconstitution of the entire capital that he utilizes and the value of the wages, the interest and the rent which he pays as well as profits belonging to him'. Schumpeter (1934) defined it as 'the process that introduces new combinations in the market'.
Hisrich and Peters (2006) defined an entrepreneur as 'one who brings resources, labour, materials, and other assets into combinations that make their value greater than before, and also, one who introduces changes, innovations, and a new order'. Austin et al (2006), Mitchell et al (2002) defined entrepreneurship as 'the opportunistic pursuit of economic wealth through creative initiatives of the individuals operating within a certain environment constrained by limited tangible resources'.

Most developed economies attribute their success to the vibrant entrepreneurs being churned out by the education system of their countries. Education should promote entrepreneurial attitude and capability among the youth enabling them to start new ventures which in turn mitigates unemployment in any country. Formal education and training can play a vital role in enhancing entrepreneurship in the country by increasing the number of individuals who start their own enterprise. This study focuses on exploring the factors which influence the entrepreneurial intent of students. Efforts can be taken to nurture the entrepreneurial spirit when they are in college which will in turn motivate them to consider entrepreneurship as a career choice. 
Graduate entrepreneurship is increasingly being seen as an important source of competitiveness, growth and economic development. It is widely acknowledged that it is important to encourage more graduates to pursue an entrepreneurial career path, that is, to start-up their own businesses. An individual with entrepreneurial education is capable of creating higher profits from entrepreneurial ventures. Graduates with an entrepreneurship major are more likely to start new businesses and have stronger entrepreneurial intentions than other graduates. Lack of entrepreneurial education leads to low level of entrepreneurial intentions of students. Psychological characteristics (e.g. risk taking propensity and entrepreneurial self-efficacy), together with developed skills and abilities, influence entrepreneurial intentions.

Entrepreneurship involves an intentional process and individuals make conscious choices and decisions which is a purposeful planned behaviour. Entrepreneurial intention helps to explain the reasons as to why entrepreneurs decide to start a business (Krueger et al., 2000). Students' intentions are significantly related to their personality traits, attitude toward entrepreneurship, perceived support and perceived barriers. Selecting and training potential entrepreneurial students to join those programs/activities based on their traits are also important. Educational institutions can have an extensive impact on the students career choices and universities can play and active role in creating a conducive environment for enhancing the interest in entrepreneurship.

\section{REVIEW OF LITERATURE}

Survey of various literature revealed that factors which affect the intention for founding one's own business can be personality factors and contextual factors. The variables taken for this study are 'risk taking propensity', 'internal locus of control', 'attitude towards entrepreneurship', 'entrepreneurial intent', 'perceived barriers' and 'perceived support' factors. Definition of the variables and the indicators used to measure each construct are given below:

\section{A. Risk Taking Propensity}

'Risk Taking Propensity' can be explained as an individual's orientation towards taking chances in any decision-making scenario. Risk taking is one of the personality traits which distinguish an entrepreneur from others who do not wish to start their own business. The entrepreneur has to take risks because decisions are made in uncertain situations (Knight, 1921). Therefore, entrepreneurs exhibit high risk-taking propensity (Stewart and Roth, 2004). A more positive attitude toward risk, leads to stronger entrepreneurial intentions( Douglas and Shepherd,2002). Risk tolerance plays an important role in the decision to become an entrepreneur. Less risk-averse individuals are more likely to start their own business (Kan and Tsai, 2006).Thus 'Risk Taking Propensity' has been considered as an important trait in the personality of entrepreneurs. It has been measured by adopting the item scale developed by Hisrich and Peters (1995).The indicators are measured using five point Likert scale ranging from 5 (very accurate) to1(very inaccurate) .

\section{B. Internal Locus of Control}

Locus of control theory developed by Rotter in 1966 is an attribute that indicates the sense of control that a person has over life (Hisrich and Peters, 1995). Locus of control refers to the individuals' overall belief in the power of their own actions across a variety of situations (Boyd and Vozikis, 1994). Gasse (1985) found that entrepreneurs often possess a greater 'Internal Locus of Control' than the general population. Individuals possessing an 'Internal Locus of Control' believe they are in control of future events and outcomes as a result of their own actions (Cromie, 2000) .Research studies have included locus of control as a construct to measure personality traits of respondents (Bonnett and Furnham, 199; Shapero, 1975; Chell et al. 1991; Cromie and Johns, 1982). Individuals with internal locus of control will have a positive attitude towards entrepreneurship which has a direct impact on entrepreneurial intention. 'Internal Locus of Control' for the present study has been measured by adopting the item scale developed by Hisrich and Peters in 1995.The indicators are measured using five point Likert scale ranging from 5 (very accurate) to1(very inaccurate) . 


\section{Attitude towards Entrepreneurship}

Attitudes are defined as beliefs and perceptions regarding the personal desirability of performing the behavior, which are in turn related to expectations regarding the personal impact of outcomes resulting from that behavior (Ajzen, 1991).If an individual displays 'high' attitude towards self-employment, it indicates that the person is more inclined to be self employed than to take up organizational employment (Kolvereid, 1996). Individual's personality traits are considered to have a direct effect on 'Attitude towards Entrepreneurship', which acts as a moderating variable between 'Entrepreneurial Intent' and 'Personality Traits' of the respondents in the 'Entrepreneurial Intention' model. 'Attitude towards Entrepreneurship' for the present study has been measured by adapting the item scale developed by Luthje and Franke, (2003). The indicators are measured using a five point Likert scale ranging from 5 (very accurate) to1(very inaccurate) .

\section{Perceived Support and Perceived Barriers to Entrepreneurship}

Contextual factors influence a person's entrepreneurial intention. Environmental factors refer to those environmental attributes that surround the individual (Grundsten, 2004). According to Luthje and Franke (2003), environmental factors can facilitate or impede entrepreneurial activities and play an important role in the formation of an individual's intention to create a new venture. Positive attitudes of the surrounding community concerning entrepreneurship are likely to increase one's desire to engage in entrepreneurial activities. Specifically, individuals who experience a positive view on entrepreneurship among their immediate contacts are more likely to have greater intention to become entrepreneurs. The items to measure 'Perceived Support ' and 'Perceived Barriers' has been developed based on study of various research articles related to entrepreneurship. The indicators are measured using a five point Likert scale ranging from 5 (to a very great extent) to1 (to a small extent).

\section{E. Entrepreneurial Intent}

Intention is defined as a state of mind directing a person's attention and action towards development and the implementation of new business concepts which leads to self-employment ( Bird, 1988). It can be seen as the intention of a person to perform new venture creation behavior or action (Grundsten, 2004). Individuals involved in new venture creation, think about it and engage in formal planning before staring their enterprise. Intentionality precedes the behavior and is considered to be a predictor and early indicator of entrepreneurial activities in the future (Krueger and Casrud, 1993; Krueger,Reilley and Carsrud,2000). Since entrepreneurship represents planned, intentional behavior (Bird, 1988; Krueger and Brazeal, 1994), and is based on the fact that intention is said to precede action (Ajzen, 1991), entrepreneurial intention has been studied as the primary dependent variable which influences a person to found his own business. In the present study, the items to measure 'Entrepreneurial Intent' have been adopted from Entrepreneurial Intention Questionnaire (EIQ) developed by Linan and Chen (2000) and Luthje and Franke, (2003). The indicators are measured using a five point Likert scale ranging from 5 (strongly agree) to 1 (strongly disagree).

\section{OBJECTIVES OF THE STUDY}

1. To determine if there is any significant difference in the personality traits of the respondents such as risk taking propensity, internal locus of control and entrepreneurial attitude due to demographic variables.

2. To find out the influence of contextual factors such as perceived support and perceived barriers on entrepreneurial intention of the respondents due to demographic variables.

\section{RESEARCH METHODOLOGY}

\section{A. Research Design and Data Collection Method}

The research design is empirical in nature and the study was conducted in two stages, with pilot testing and validation followed by the main study. Primary and secondary methods of data collection were used. Secondary data for this study was obtained from studies done by past researchers, websites and online databases. Primary data collection method was done through structured questionnaire which was administered by the researcher in person to the respondents of the study. Careful pilot testing of the draft questionnaire was 
conducted to confirm its reliability and validity. The respondents were asked to review the questionnaire and fill in their responses. Based on their feedback, the questionnaire was improved, after which the same was distributed to the students in the select Arts and Science Colleges affiliated to the University of Madras after getting approval from the relevant authorities

\section{B. Sampling Plan}

The population comprises of Arts and Science colleges affiliated to University of Madras in Chennai city. From the targeted population, a sample size of 600 respondents was selected for the study. Two stage stratified random sampling technique was used for selecting the respondents. The first stage involved selection of the type of college namely, 'Government Arts and Science' colleges which are run by the Government department, 'Government Aided Autonomous Arts and Science' colleges which are managed by trusts with financial aid given by the Government and 'Self- financing Arts and Science' colleges. The second stage was based on the level of education of the respondents' namely undergraduate and postgraduate levels. 300 undergraduate students and 300 post graduate students pursuing their final year in college were selected to constitute the sample.

\section{Statistical Techniques Used for Data Analysis}

The collected data is analysed using t-test and one way ANOVA. T-test is a parametric test to determine the statistical significance between a sample distribution mean and a population parameter. Anova tests the null hypothesis that the means of several independent populations are equal.

\section{Limitations of the Study}

- The study was restricted to only Arts and Science colleges affiliated to University of Madras in Chennai and hence the inferences drawn are to be carefully considered for generalisation.

- Items measured under each construct can be reexamined for other disciplines of study or other types of universities.

- The study has been conducted considering the existing status of students studying in colleges which may be subject to change in future.
- This study is limited by its cross-sectional data; students are observed only at a single point in time, not across time.

- Since the study was restricted to academia, caution must be exercised in generalizing the results in other fields.

\section{DATA ANALYSIS}

\section{A. INDEPENDENT T- TEST}

HYPOTHESIS I: There is no significant difference between male and female students with respect to intention to become entrepreneur.

Table 1: Student t-test for significant difference between male and female students with respect to intention to become entrepreneur.

\begin{tabular}{|c|c|c|c|c|c|c|}
\hline \multirow{2}{*}{$\begin{array}{l}\text { Factors of } \\
\text { Entrepreneu } \\
\text { rial } \\
\text { Intention }\end{array}$} & \multicolumn{2}{|c|}{ Male } & \multicolumn{2}{|c|}{ Female } & \multirow{2}{*}{$\begin{array}{c}\mathrm{t} \\
\text { value }\end{array}$} & \multirow{2}{*}{$\begin{array}{c}P \\
\text { value }\end{array}$} \\
\hline & Mean & S.D & Mean & S.D & & \\
\hline $\begin{array}{l}\text { Risk taking } \\
\text { Propensity }\end{array}$ & 27.43 & 5.37 & 25.22 & 4.72 & 5.362 & $\underset{* *}{<0.001}$ \\
\hline $\begin{array}{l}\text { Internal Locus } \\
\text { of Control }\end{array}$ & 25.39 & 4.95 & 25.04 & 4.51 & 0.912 & 0.362 \\
\hline $\begin{array}{l}\text { Attitude } \\
\text { towards } \\
\text { Entrepreneurs } \\
\text { hip }\end{array}$ & 10.62 & 4.10 & 9.20 & 4.15 & 4.203 & $<0.001$ \\
\hline $\begin{array}{l}\text { Entrepreneuria } \\
\text { I Intent }\end{array}$ & 21.78 & 5.80 & 19.67 & 6.14 & 4.325 & $\underset{* *}{<0.001}$ \\
\hline $\begin{array}{l}\text { Perceived } \\
\text { Support }\end{array}$ & 46.91 & 7.49 & 46.37 & 7.48 & 0.883 & 0.378 \\
\hline $\begin{array}{l}\text { Perceived } \\
\text { Barriers }\end{array}$ & 47.51 & 10.82 & 47.67 & 10.22 & 0.182 & 0.855 \\
\hline
\end{tabular}

Note: ** Denotes significant at $1 \%$ level

HYPOTHESIS II: There is no significant difference between students pursuing under graduation and post graduation courses with respect to intention to become entrepreneur.

Table 2: Student t-test for significant difference between students pursuing under graduation and post graduation courses with respect to intention to become entrepreneur.

\begin{tabular}{|c|c|c|c|c|c|c|}
\hline \multirow{2}{*}{$\begin{array}{l}\text { Factors of } \\
\text { Entrepreneurial } \\
\text { Intention }\end{array}$} & \multicolumn{2}{|c|}{ U.G } & \multicolumn{2}{|c|}{ P.G } & \multirow{2}{*}{$\begin{array}{c}t \\
\text { value }\end{array}$} & \multirow{2}{*}{$\begin{array}{c}P \\
\text { value }\end{array}$} \\
\hline & Mean & S.D & $\begin{array}{l}\text { Me } \\
\text { an }\end{array}$ & S.D & & \\
\hline $\begin{array}{l}\text { Risk taking } \\
\text { Propensity }\end{array}$ & 26.84 & 5.02 & $\begin{array}{l}25 . \\
72\end{array}$ & 5.25 & 2.672 & 0.008 \\
\hline $\begin{array}{l}\text { Internal Locus of } \\
\text { Control }\end{array}$ & 25.55 & 4.73 & $\begin{array}{l}24 . \\
87\end{array}$ & 4.71 & 1.755 & 0.080 \\
\hline $\begin{array}{l}\text { Attitude towards } \\
\text { Entrepreneurship }\end{array}$ & 10.38 & 4.01 & $\begin{array}{c}9.3 \\
9\end{array}$ & 4.30 & 2.897 & 0.004 \\
\hline $\begin{array}{l}\text { Entrepreneurial } \\
\text { Intent }\end{array}$ & 21.23 & 5.71 & $\begin{array}{l}20 . \\
14\end{array}$ & 6.37 & 2.200 & $\begin{array}{c}0.028 \\
*\end{array}$ \\
\hline $\begin{array}{l}\text { Perceived } \\
\text { Support }\end{array}$ & 46.07 & 7.14 & $\begin{array}{l}47 . \\
18 \\
\end{array}$ & 7.79 & 1.814 & 0.070 \\
\hline $\begin{array}{l}\text { Perceived } \\
\text { Barriers }\end{array}$ & 47.40 & 10.11 & $\begin{array}{l}47 . \\
78\end{array}$ & $\begin{array}{c}10.8 \\
9\end{array}$ & 0.443 & 0.658 \\
\hline
\end{tabular}


Note: ** Denotes significant at $1 \%$ level. *Denotes significant at 0.05 $\%$ level

HYPOTHESIS III: There is no significant difference between students from rural background and urban background with respect to intention to become entrepreneur.

Table 3: Student t-test for significant difference between students from rural background and urban background with respect to intention to become entrepreneur.

\begin{tabular}{|c|c|c|c|c|c|c|}
\hline \multirow{2}{*}{$\begin{array}{l}\text { Factors of } \\
\text { Entrepreneurial } \\
\text { Intention }\end{array}$} & \multicolumn{2}{|c|}{ RURAL } & \multicolumn{2}{|c|}{ URBAN } & \multirow{2}{*}{$\begin{array}{c}t \\
\text { value }\end{array}$} & \multirow{2}{*}{$\begin{array}{c}P \\
\text { value }\end{array}$} \\
\hline & Mean & S.D & Mean & S.D & & \\
\hline $\begin{array}{l}\text { Risk taking } \\
\text { Propensity }\end{array}$ & 27.01 & 5.15 & 26.04 & 5.15 & 2.006 & $0.045^{*}$ \\
\hline $\begin{array}{l}\text { Internal Locus of } \\
\text { Control }\end{array}$ & 25.21 & 5.13 & 25.21 & 4.60 & 0.19 & 0.985 \\
\hline $\begin{array}{l}\text { Attitude towards } \\
\text { Entrepreneurship }\end{array}$ & 10.19 & 4.10 & 9.78 & 4.21 & 1.042 & 0.298 \\
\hline $\begin{array}{l}\text { Entrepreneurial } \\
\text { Intent }\end{array}$ & 21.19 & 5.48 & 20.52 & 6.25 & 1.171 & 0.242 \\
\hline Perceived Support & 46.68 & 8.11 & 46.61 & 7.28 & 0.096 & 0.923 \\
\hline Perceived Barriers & 48.23 & 10.17 & 47.38 & 10.61 & 0.851 & 0.395 \\
\hline
\end{tabular}

HYPOTHESIS IV: There is no significant difference between students from North India and South India with respect to intention to become entrepreneur.

Table 4: Student t-test for significant difference students from North India and South India with respect to intention to become entrepreneur.

\begin{tabular}{|l|c|c|c|c|c|c|}
\hline $\begin{array}{l}\text { Factors of } \\
\text { Entrepreneurial } \\
\text { Intention }\end{array}$ & \multicolumn{2}{|c|}{$\begin{array}{c}\text { NORTH } \\
\text { INDIAN }\end{array}$} & \multicolumn{2}{|c|}{$\begin{array}{c}\text { SOUTH } \\
\text { INDIAN }\end{array}$} & $\begin{array}{c}\mathbf{t} \\
\text { value }\end{array}$ & $\begin{array}{c}\mathbf{P} \\
\text { value }\end{array}$ \\
\cline { 2 - 7 } & Mean & S.D & Mean & S.D & & \\
\hline $\begin{array}{l}\text { Risk taking } \\
\text { Propensity }\end{array}$ & 26.75 & 4.98 & 26.23 & 5.18 & 0.743 & 0.458 \\
\hline $\begin{array}{l}\text { Internal Locus of } \\
\text { Control }\end{array}$ & 25.33 & 4.55 & 25.19 & 4.75 & 0.216 & 0.829 \\
\hline Attitude & 10.53 & 4.50 & 9.81 & 4.14 & 1.266 & 0.206 \\
\hline $\begin{array}{l}\text { Entrepreneurial } \\
\text { Intent }\end{array}$ & 22.28 & 6.20 & 20.51 & 6.03 & 2.160 & 0.031 \\
\hline $\begin{array}{l}\text { Perceived } \\
\text { Support }\end{array}$ & 46.98 & 6.46 & 46.59 & 7.60 & 0.389 & 0.698 \\
\hline $\begin{array}{l}\text { Perceived } \\
\text { Barriers }\end{array}$ & 47.13 & 8.79 & 47.64 & 10.62 & 0.357 & 0.721 \\
\hline
\end{tabular}

HYPOTHESIS V: There is no significant difference between students having work experience and students without work experience with respect to intention to become entrepreneur.
Table 5: Student T- test for significant difference between students having work experience and students without work experience with respect to intention to become entrepreneur.

\begin{tabular}{|c|c|c|c|c|c|c|}
\hline \multirow{3}{*}{$\begin{array}{l}\text { Factors of } \\
\text { Entrepreneu } \\
\text { rial } \\
\text { Intention }\end{array}$} & \multicolumn{4}{|c|}{ Work experience } & \multirow{3}{*}{$\begin{array}{c}t \\
\text { valu } \\
e\end{array}$} & \multirow{3}{*}{$P$ value } \\
\hline & \multicolumn{2}{|c|}{ YES } & \multicolumn{2}{|c|}{ NO } & & \\
\hline & Mean & S.D & Mean & S.D & & \\
\hline $\begin{array}{l}\text { Risk taking } \\
\text { Propensity }\end{array}$ & 27.81 & 5.23 & 25.62 & 4.99 & 4.85 & $\underset{*}{<0.001^{*}}$ \\
\hline $\begin{array}{l}\text { Internal } \\
\text { Locus of } \\
\text { Control }\end{array}$ & 26.05 & 4.72 & 24.84 & 4.69 & 2.88 & $\underset{*}{<0.001^{*}}$ \\
\hline Attitude & 10.94 & 3.85 & 9.43 & 4.24 & 4.13 & $\underset{*}{<0.001^{*}}$ \\
\hline $\begin{array}{l}\text { Entrepreneur } \\
\text { ial Intent }\end{array}$ & 22.37 & 5.53 & 19.95 & 6.15 & 4.55 & $\underset{*}{<0.001^{*}}$ \\
\hline $\begin{array}{l}\text { Perceived } \\
\text { Support }\end{array}$ & 47.88 & 7.21 & 46.08 & 7.55 & 2.71 & $\begin{array}{c}<0.001^{*} \\
*\end{array}$ \\
\hline $\begin{array}{l}\text { Perceived } \\
\text { Barriers }\end{array}$ & 47.75 & $\begin{array}{c}10.9 \\
9\end{array}$ & 47.53 & 10.30 & 0.24 & 0.809 \\
\hline
\end{tabular}

Note: ** Denotes significant at $1 \%$ level

HYPOTHESIS VI: There is no significant difference between students from joint family and nuclear family with respect to intention to become entrepreneur.

Table 6: Student t test for significant difference between students from joint family and nuclear family with respect to intention to become entrepreneur.

\begin{tabular}{|l|l|l|l|l|c|c|}
\hline Factors of & \multicolumn{4}{|c|}{ Type of Family } & \multicolumn{1}{c|}{\begin{tabular}{c}
$\mathbf{P}$ \\
Entrepreneuria \\
\cline { 2 - 5 } I Intention
\end{tabular}} & \multicolumn{3}{|c|}{ JOINT } & \multicolumn{2}{c|}{ NUCLEAR } & value & value \\
\cline { 2 - 5 } & Mean & S.D & Mean & S.D & & \\
\hline $\begin{array}{l}\text { Risk taking } \\
\text { Propensity }\end{array}$ & 26.60 & 4.82 & 26.18 & 5.26 & 0.83 & 0.407 \\
\hline $\begin{array}{l}\text { Internal Locus } \\
\text { of Control }\end{array}$ & 25.38 & 4.63 & 25.15 & 4.76 & 0.50 & 0.617 \\
\hline $\begin{array}{l}\text { Attitude towards } \\
\text { Entrepreneurshi } \\
p\end{array}$ & 10.32 & 4.27 & 9.75 & 4.15 & 1.41 & 0.159 \\
\hline $\begin{array}{l}\text { Entrepreneurial } \\
\text { Intent }\end{array}$ & 21.07 & 6.03 & 20.56 & 6.08 & 0.87 & 0.386 \\
\hline $\begin{array}{l}\text { Perceived } \\
\text { Support }\end{array}$ & 46.76 & 6.92 & 46.98 & 7.66 & 0.24 & 0.811 \\
\hline $\begin{array}{l}\text { Perceived } \\
\text { Barriers }\end{array}$ & 48.67 & 9.55 & 47.26 & 10.7 & 1.39 & 0.163 \\
\hline
\end{tabular}

ONE WAY ANOVA

HYPOTHESIS VII: There is no significant difference between students pursuing under graduate course of study with respect to intention to become entrepreneurs. 
Table 7: ANOVA for significant difference between students pursuing under graduate course of study with respect to impact of factors on intention to become entrepreneur.

\begin{tabular}{|c|c|c|c|c|c|c|}
\hline \multirow{2}{*}{$\begin{array}{l}\text { Factors of } \\
\text { Entrepreneurial } \\
\text { Intention }\end{array}$} & \multicolumn{4}{|c|}{ Course of Study-UG } & \multirow{2}{*}{$\begin{array}{c}F \\
\text { value }\end{array}$} & \multirow{2}{*}{$\begin{array}{c}P \\
\text { value }\end{array}$} \\
\hline & BA & B.Sc & BBA & B.Com & & \\
\hline $\begin{array}{l}\text { Risk taking } \\
\text { Propensity }\end{array}$ & $\begin{array}{l}27.68 \\
(4.35)\end{array}$ & $\begin{array}{c}26.89 \\
(4.817) \\
\end{array}$ & $\begin{array}{c}25.85 \\
(4.906) \\
\end{array}$ & $\begin{array}{r}26.88 \\
(5.34) \\
\end{array}$ & 0.669 & 0.572 \\
\hline $\begin{array}{l}\text { Internal Locus of } \\
\text { Control }\end{array}$ & $\begin{array}{l}24.48 \\
(4.47)\end{array}$ & $\begin{array}{l}25.65 \\
(4.94)\end{array}$ & $\begin{array}{l}26.06 \\
(4.34)\end{array}$ & $\begin{array}{l}25.53 \\
(4.73)\end{array}$ & 0.571 & 0.635 \\
\hline $\begin{array}{l}\text { Attitude towards } \\
\text { Entrepreneurship }\end{array}$ & $\begin{array}{l}10.72 \\
(3.46)\end{array}$ & $\begin{array}{l}10.40 \\
(4.19)\end{array}$ & $\begin{array}{l}10.64 \\
(3.75)\end{array}$ & $\begin{array}{l}10.22 \\
(4.06)\end{array}$ & 0.168 & 0.918 \\
\hline $\begin{array}{l}\text { Entrepreneurial } \\
\text { Intent }\end{array}$ & $\begin{array}{l}20.16 \\
(6.13)\end{array}$ & $\begin{array}{l}20.88 \\
(5.93)\end{array}$ & $\begin{array}{l}22.52 \\
(5.25)\end{array}$ & $\begin{array}{l}21.41 \\
(5.53)\end{array}$ & 1.039 & 0.375 \\
\hline
\end{tabular}

\begin{tabular}{|l|l|l|l|l|l|l|}
\hline $\begin{array}{l}\text { Perceived } \\
\text { Support }\end{array}$ & $\begin{array}{l}47.64 \\
(6.87)\end{array}$ & $\begin{array}{l}45.74 \\
(7.63)\end{array}$ & $\begin{array}{l}44.24 \\
(6.57)\end{array}$ & $\begin{array}{l}46.53 \\
(6.85)\end{array}$ & 1.384 & 0.248 \\
\hline $\begin{array}{l}\text { Perceived } \\
\text { Barriers }\end{array}$ & $51.16^{\mathrm{c}}$ & $49.22^{\mathrm{bc}}$ & $42.82^{\mathrm{a}}$ & $46.26^{\mathrm{ab}}$ & & \\
& $(7.48)$ & $(10.42)$ & $(8.45)$ & $(10.16)$ & 5.411 & $0.001^{* *}$ \\
\hline
\end{tabular}

Note: $\quad$ 1.The value within bracket refers to standard deviation. 2. ${ }^{* *}$ denotes significant at $1 \%$ level.

3. Different alphabet between under graduate course of study of the respondents denotes significant at $5 \%$ level using Duncan Multiple Range Test (DMRT).

HYPOTHESIS VIII: There is no significant difference among post graduate student respondents with respect to intention to become entrepreneurs.

Table 8: ANOVA for significant difference between post graduate student respondents with respect to impact of factors on intention to become entrepreneur.

\begin{tabular}{|c|c|c|c|c|c|c|c|}
\hline \multirow{2}{*}{$\begin{array}{l}\text { Factors of } \\
\text { Entrepreneurial } \\
\text { Intention }\end{array}$} & \multicolumn{5}{|c|}{ Course of Study-PG } & \multirow{2}{*}{$\begin{array}{c}F \\
\text { value }\end{array}$} & \multirow[t]{2}{*}{$P$ value } \\
\hline & M.A & M.Sc & M.Com & M.B.A & M.C.A & & \\
\hline $\begin{array}{l}\text { Risk taking } \\
\text { Propensity }\end{array}$ & $\begin{array}{c}26.22^{\mathrm{bc}} \\
(5.19)\end{array}$ & $\begin{array}{l}27.60^{c} \\
(5.83)\end{array}$ & $\begin{array}{c}24.16^{\mathrm{ab}} \\
(4.98)\end{array}$ & $\begin{array}{c}24.64^{\mathrm{ab}} \\
(4.58)\end{array}$ & $\begin{array}{l}23.57^{a} \\
(3.75)\end{array}$ & 6.067 & $<0.001^{* *}$ \\
\hline $\begin{array}{l}\text { Internal Locus of } \\
\text { Control }\end{array}$ & $\begin{array}{l}25.60^{\mathrm{b}} \\
(5.18)\end{array}$ & $\begin{array}{l}25.28^{b} \\
(4.35)\end{array}$ & $\begin{array}{l}23.22^{a} \\
(4.50)\end{array}$ & $\begin{array}{l}25.18^{b} \\
(4.44)\end{array}$ & $\begin{array}{l}23.14^{a} \\
(4.30)\end{array}$ & 3.078 & $0.017^{*}$ \\
\hline $\begin{array}{l}\text { Attitude towards } \\
\text { Entrepreneurship }\end{array}$ & $\begin{array}{l}10.56^{c} \\
(3.97)\end{array}$ & $\begin{array}{l}9.89 \mathrm{bc} \\
(4.67)\end{array}$ & $\begin{array}{l}8.69 \mathrm{~b} \\
(4.50)\end{array}$ & $\begin{array}{l}9.15^{b c} \\
(3.86)\end{array}$ & $\begin{array}{l}6.51^{\mathrm{a}} \\
(3.37)\end{array}$ & 6.818 & $<0.001^{* *}$ \\
\hline $\begin{array}{l}\text { Entrepreneurial } \\
\text { Intent }\end{array}$ & $\begin{array}{l}21.96^{b} \\
(5.87)\end{array}$ & $\begin{array}{c}19.38^{a b} \\
(6.39)\end{array}$ & $\begin{array}{c}20.22^{\mathrm{ab}} \\
(6.49)\end{array}$ & $\begin{array}{c}19.75^{\mathrm{ab}} \\
(7.02)\end{array}$ & $\begin{array}{l}17.95^{\mathrm{a}} \\
(5.41) \\
\end{array}$ & 3.372 & $0.010^{\star *}$ \\
\hline $\begin{array}{l}\text { Perceived } \\
\text { Support }\end{array}$ & $\begin{array}{l}48.43^{b} \\
(7.20)\end{array}$ & $\begin{array}{l}47.96^{b} \\
(7.59)\end{array}$ & $\begin{array}{l}47.84^{b} \\
(7.80)\end{array}$ & $\begin{array}{l}47.30^{b} \\
(7.77)\end{array}$ & $\begin{array}{l}41.68^{a} \\
(7.70)\end{array}$ & 5.822 & $<0.001^{* *}$ \\
\hline $\begin{array}{l}\text { Perceived } \\
\text { Barriers }\end{array}$ & $\begin{array}{c}47.40 \\
(10.70)\end{array}$ & $\begin{array}{c}49.83 \\
(11.11)\end{array}$ & $\begin{array}{c}46.94 \\
(14.57)\end{array}$ & $\begin{array}{l}48.07 \\
(9.37)\end{array}$ & $\begin{array}{l}44.57 \\
(9.03)\end{array}$ & 1.608 & 0.172 \\
\hline
\end{tabular}

Note: 1 .The value within bracket refers to standard deviation.

2. ${ }^{* *}$ denotes significant at $1 \%$ level.

3. ${ }^{*}$ denotes significant at $5 \%$ level. 4 . Different alphabet between courses of study of the respondents denotes significant at $5 \%$ level using Duncan Multiple Range test (DMRT).

HYPOTHESIS IX: There is no significant difference between birth order of the respondents with respect to impact of factors on intention to become entrepreneurs.

Table 9: ANOVA for significant difference between birth orders of the respondents with respect to impact of factors on intention to become entrepreneur.

\begin{tabular}{|c|c|c|c|c|c|c|}
\hline \multirow{2}{*}{$\begin{array}{l}\text { Factors of } \\
\text { Entrepreneurial } \\
\text { Intention }\end{array}$} & \multicolumn{4}{|c|}{ Birth Order } & \multirow{2}{*}{$\begin{array}{c}F \\
\text { value }\end{array}$} & \multirow[t]{2}{*}{$P$ value } \\
\hline & First & Second & Third & $\begin{array}{l}\text { Fourth and } \\
\text { Above }\end{array}$ & & \\
\hline $\begin{array}{l}\text { Risk taking } \\
\text { Propensity }\end{array}$ & $\begin{array}{l}25.80 \\
(5.07)\end{array}$ & $\begin{array}{l}26.44 \\
(5.19)\end{array}$ & $\begin{array}{l}26.90 \\
(4.74)\end{array}$ & $\begin{array}{l}26.71 \\
(6.12)\end{array}$ & 1.33 & 0.26 \\
\hline Internal Locus of & 25.35 & 25.21 & 25.01 & 24.90 & 0.19 & 0.90 \\
\hline
\end{tabular}




\begin{tabular}{|c|c|c|c|c|c|c|}
\hline Control & $(4.76)$ & $(4.62)$ & $(4.34)$ & $(5.79)$ & & \\
\hline $\begin{array}{l}\text { Attitude towards } \\
\text { Entrepreneurship }\end{array}$ & $\begin{array}{c}9.90 \\
(4.16)\end{array}$ & $\begin{array}{c}9.80 \\
(4.24)\end{array}$ & $\begin{array}{c}9.99 \\
(4.10)\end{array}$ & $\begin{array}{l}10.02 \\
(4.30)\end{array}$ & 0.07 & 0.97 \\
\hline $\begin{array}{l}\text { Entrepreneurial } \\
\text { Intent }\end{array}$ & $\begin{array}{l}20.43 \\
(6.26)\end{array}$ & $\begin{array}{l}20.93 \\
(6.13)\end{array}$ & $\begin{array}{l}20.38 \\
(5.17)\end{array}$ & $\begin{array}{l}21.45 \\
(6.59)\end{array}$ & 0.59 & 0.62 \\
\hline Perceived Support & $\begin{array}{l}46.59 \\
(7.64)\end{array}$ & $\begin{array}{c}46.56 \\
(7.092)\end{array}$ & $\begin{array}{l}46.67 \\
(7.716)\end{array}$ & $\begin{array}{l}47.02 \\
(8.097)\end{array}$ & 0.05 & 0.98 \\
\hline Perceived Barriers & $\begin{array}{l}48.10 \\
(10.4)\end{array}$ & $\begin{array}{c}47.02 \\
(11.087)\end{array}$ & $\begin{array}{l}47.79 \\
(9.26)\end{array}$ & $\begin{array}{c}47.22 \\
(10.639)\end{array}$ & 0.43 & 0.72 \\
\hline
\end{tabular}

Note: $\quad$ 1.The value within bracket refers to standard deviation.

HYPOTHESIS X: There is no significant difference between occupation of father with respect to impact of factors on intention to become entrepreneurs.

Table 10: ANOVA for significant difference between occupation of father with respect to impact of factors on intention to become entrepreneur.

\begin{tabular}{|c|c|c|c|c|c|c|c|c|}
\hline \multirow{2}{*}{$\begin{array}{l}\text { Factors of } \\
\text { Entrepreneurial } \\
\text { Intention }\end{array}$} & \multicolumn{6}{|c|}{ Occupation of Father } & \multirow{2}{*}{$\begin{array}{c}F \\
\text { value }\end{array}$} & \multirow{2}{*}{$\begin{array}{c}P \\
\text { value }\end{array}$} \\
\hline & 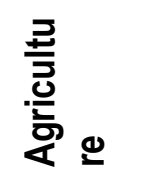 & 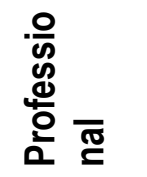 & 호응 을 & 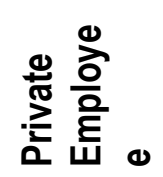 & 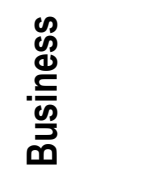 & $\begin{array}{l}\frac{n}{d} \\
\frac{c}{0}\end{array}$ & & \\
\hline $\begin{array}{l}\text { Risk taking } \\
\text { Propensity }\end{array}$ & $\begin{array}{l}26.4 \\
(5.61)\end{array}$ & $\begin{array}{l}25.7 \\
(5.07)\end{array}$ & $\begin{array}{l}26 \\
(5)\end{array}$ & $\begin{array}{l}25.5 \\
(5.19)\end{array}$ & $\begin{array}{l}26.78 \\
(4.76)\end{array}$ & $\begin{array}{l}27.50 \\
(6.30)\end{array}$ & 1.351 & 0.241 \\
\hline $\begin{array}{l}\text { Internal Locus of } \\
\text { Control }\end{array}$ & $\begin{array}{l}24.76 \mathrm{ab} \\
(5.90)\end{array}$ & $\begin{array}{l}24.06^{a} \\
(4.78)\end{array}$ & $\begin{array}{l}24.86^{a b} \\
(4.75)\end{array}$ & $\begin{array}{l}25.12^{\mathrm{ab}} \\
(4.69)\end{array}$ & $\begin{array}{l}25.83^{b c} \\
(4.12)\end{array}$ & $\begin{array}{l}27.08^{c} \\
(3.98)\end{array}$ & 2.976 & $0.012^{*}$ \\
\hline $\begin{array}{l}\text { Attitude towards } \\
\text { Entrepreneurship }\end{array}$ & $\begin{array}{l}9.91 \mathrm{ab} \\
(4.55)\end{array}$ & $\begin{array}{l}9.18^{a} \\
(3.70)\end{array}$ & $\begin{array}{l}9.32^{\mathrm{a}} \\
(4.45)\end{array}$ & $\begin{array}{l}9.27 a \\
(4.19)\end{array}$ & $\begin{array}{l}10.75^{b} \\
(3.98)\end{array}$ & $\begin{array}{l}11.00^{\mathrm{b}} \\
(3.51)\end{array}$ & 3.443 & $0.004^{* *}$ \\
\hline $\begin{array}{l}\text { Entrepreneurial } \\
\text { Intent }\end{array}$ & $\begin{array}{l}20.61^{\mathrm{ab}} \\
(6.30)\end{array}$ & $\begin{array}{l}20.23^{a b} \\
(5.93)\end{array}$ & $\begin{array}{l}19.37^{a} \\
(5.98)\end{array}$ & $\begin{array}{l}19.98^{\mathrm{ab}} \\
(6.47)\end{array}$ & $\begin{array}{l}22.08^{\mathrm{b}} \\
(5.77)\end{array}$ & $\begin{array}{l}21.89^{b} \\
(5.15)\end{array}$ & 3.840 & $0.002^{* *}$ \\
\hline $\begin{array}{l}\text { Perceived } \\
\text { Support }\end{array}$ & $\begin{array}{l}45.84 \mathrm{ab} \\
(8.74)\end{array}$ & $\begin{array}{l}46.31 \mathrm{ab} \\
(7.30)\end{array}$ & $\begin{array}{l}46.03^{a b} \\
(7.77)\end{array}$ & $\begin{array}{l}45.15^{\mathrm{a}} \\
(7.27)\end{array}$ & $\begin{array}{l}47.79 \mathrm{bc} \\
(6.82)\end{array}$ & $\begin{array}{l}49.72^{c} \\
(6.41)\end{array}$ & 3.316 & $0.006^{* *}$ \\
\hline $\begin{array}{l}\text { Perceived } \\
\text { Barriers }\end{array}$ & $\begin{array}{l}48.24 \\
(9.97)\end{array}$ & $\begin{array}{l}47.68 \\
(9.52)\end{array}$ & $\begin{array}{l}48.33 \\
(10.04)\end{array}$ & $\begin{array}{l}46.67 \\
(10.31)\end{array}$ & $\begin{array}{l}46.49 \\
(11.85)\end{array}$ & $\begin{array}{l}51.33 \\
(8.06)\end{array}$ & 1.656 & 0.143 \\
\hline
\end{tabular}

Note: $\quad$ 1.The value within bracket refers to standard deviation. $2 .{ }^{* *}$ denotes significant at $1 \%$ level. $3 .{ }^{*}$ denotes significant at $5 \%$ level. 4 . Different alphabet between occupation of father denotes significant at $5 \%$ level using Duncan Multiple Range Test (DMRT).

HYPOTHESIS XI: There is no significant difference between occupation of mother with respect to impact of factors on intention to become entrepreneurs. 
Table 11: ANOVA for significant difference between occupation of mother with respect to impact of factors on intention to become entrepreneur.

\begin{tabular}{|c|c|c|c|c|c|c|c|c|}
\hline \multirow{2}{*}{$\begin{array}{ll}\text { Factors } & \text { of } \\
\text { Entrepreneurial } \\
\text { Intention }\end{array}$} & \multicolumn{6}{|c|}{ Occupation ofMother } & \multirow[t]{2}{*}{$F$ value } & \multirow[t]{2}{*}{ P value } \\
\hline & 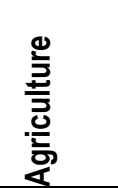 & 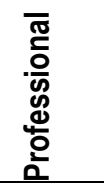 & 苍 客 & 胥 & 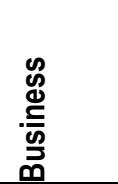 & 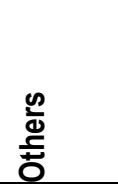 & & \\
\hline $\begin{array}{ll}\text { Risk } & \text { taking } \\
\text { Propensity } & \end{array}$ & $\begin{array}{l}28.00 \\
(5.60)\end{array}$ & $\begin{array}{l}26.0 \\
(4.4)\end{array}$ & $\begin{array}{l}24.83 \\
(4.83)\end{array}$ & $\begin{array}{l}27.77 \\
(4.46)\end{array}$ & $\begin{array}{l}26.74 \\
(4.84)\end{array}$ & $\begin{array}{l}26.14 \\
(5.21)\end{array}$ & 1.867 & 0.09 \\
\hline $\begin{array}{l}\text { Internal Locus of } \\
\text { Control }\end{array}$ & $\begin{array}{l}25.24 \\
(5.29)\end{array}$ & $\begin{array}{l}24.97 \\
(4.48)\end{array}$ & $\begin{array}{l}23.77 \\
(4.64)\end{array}$ & $\begin{array}{l}27.62 \\
(3.70)\end{array}$ & $\begin{array}{l}25.00 \\
(5.31)\end{array}$ & $\begin{array}{l}25.19 \\
(4.70)\end{array}$ & 1.946 & 0.08 \\
\hline $\begin{array}{l}\text { Attitude towards } \\
\text { Entrepreneurship }\end{array}$ & $\begin{array}{l}11.00 \\
(3.91)\end{array}$ & $\begin{array}{l}9.21 \\
(3.76)\end{array}$ & $\begin{array}{l}9.40 \\
(4.09)\end{array}$ & $\begin{array}{l}10.69 \\
(3.64)\end{array}$ & $\begin{array}{l}9.89 \\
(3.13)\end{array}$ & $\begin{array}{l}9.83 \\
(4.30)\end{array}$ & 1.011 & 0.41 \\
\hline Entrepreneurial Intent & $\begin{array}{l}21.05 \\
(5.18) \\
\end{array}$ & $\begin{array}{l}20.03 \\
(5.10) \\
\end{array}$ & $\begin{array}{l}19.40 \\
(6.02) \\
\end{array}$ & $\begin{array}{l}18.50 \\
(6.77) \\
\end{array}$ & $\begin{array}{l}21.21 \\
(4.25) \\
\end{array}$ & $\begin{array}{l}20.89 \\
(6.22) \\
\end{array}$ & 1.184 & 0.31 \\
\hline Perceived Support & $\begin{array}{l}46.71 \\
(8.85)\end{array}$ & $\begin{array}{l}44.82 \\
(6.74)\end{array}$ & $\begin{array}{l}44.47 \\
(7.55)\end{array}$ & $\begin{array}{l}48.19 \\
(6.70)\end{array}$ & $\begin{array}{l}47.47 \\
(8.25)\end{array}$ & $\begin{array}{l}46.77 \\
(7.41)\end{array}$ & 1.208 & 0.30 \\
\hline Perceived Barriers & $\begin{array}{l}50.95 \\
(10.47)\end{array}$ & $\begin{array}{l}47.24 \\
(9.47)\end{array}$ & $\begin{array}{l}45.47 \\
(8.92)\end{array}$ & $\begin{array}{l}51.08 \\
(9.94)\end{array}$ & $\begin{array}{l}50.32 \\
(11.08)\end{array}$ & $\begin{array}{l}47.17 \\
(10.62)\end{array}$ & 2.024 & 0.07 \\
\hline
\end{tabular}

Note: $\quad$ 1.The value within bracket refers to standard deviation.

HYPOTHESIS XII: There is no significant difference between monthly family income of the respondents with respect to impact of factors on intention to become entrepreneurs.

Table 12: ANOVA for significant difference between monthly family income of the respondents with respect to impact of factors on intention to become entrepreneur.

\begin{tabular}{|c|c|c|c|c|c|c|c|}
\hline \multirow{2}{*}{$\begin{array}{l}\text { Factors of Entrepreneurial } \\
\text { Intention }\end{array}$} & \multicolumn{5}{|c|}{ Monthly family income in Rupees } & \multirow[t]{2}{*}{ F value } & \multirow[t]{2}{*}{$P$ value } \\
\hline & $\begin{array}{l}\text { Below } \\
10000\end{array}$ & $10000-20000$ & $\begin{array}{l}20000- \\
30000\end{array}$ & $30000-40000$ & Above 40000 & & \\
\hline Risk taking Propensity & $\begin{array}{l}26.74 \\
(5.62)\end{array}$ & $\begin{array}{l}26.25 \\
(5.02)\end{array}$ & $\begin{array}{l}26.40 \\
(5.06)\end{array}$ & $\begin{array}{l}25.58 \\
(4.53)\end{array}$ & $\begin{array}{l}26.17 \\
(5.39)\end{array}$ & 0.759 & 0.553 \\
\hline Internal Locus of Control & $\begin{array}{l}25.17 \\
(5.16)\end{array}$ & $\begin{array}{l}25.03 \\
(4.46)\end{array}$ & $\begin{array}{l}24.90 \\
(4.43)\end{array}$ & $\begin{array}{l}25.31 \\
(4.62)\end{array}$ & $\begin{array}{l}25.87 \\
(4.94)\end{array}$ & 0.615 & 0.652 \\
\hline $\begin{array}{l}\text { Attitude towards } \\
\text { Entrepreneurship }\end{array}$ & $\begin{array}{l}10.03 \\
(3.88)\end{array}$ & $\begin{array}{c}9.63 \\
(4.31)\end{array}$ & $\begin{array}{l}9.98 \\
(4.63)\end{array}$ & $\begin{array}{c}9.47 \\
(3.74)\end{array}$ & $\begin{array}{l}10.44 \\
(4.36)\end{array}$ & 0.822 & 0.512 \\
\hline Entrepreneurial Intent & $\begin{array}{l}20.76 \\
(6.14)\end{array}$ & $\begin{array}{l}20.68 \\
(5.74)\end{array}$ & $\begin{array}{l}20.38 \\
(5.99)\end{array}$ & $\begin{array}{l}20.97 \\
(5.84)\end{array}$ & $\begin{array}{l}20.61 \\
(6.92)\end{array}$ & 0.129 & 0.972 \\
\hline Perceived Support & $\begin{array}{l}47.03 \\
(6.68)\end{array}$ & $\begin{array}{l}45.82 \\
(7.82)\end{array}$ & $\begin{array}{l}46.57 \\
(7.59)\end{array}$ & $\begin{array}{l}47.81 \\
(6.53)\end{array}$ & $\begin{array}{l}46.20 \\
(8.85)\end{array}$ & 1.253 & 0.287 \\
\hline Perceived Barriers & $\begin{array}{c}48.95 \\
(10.38)\end{array}$ & $\begin{array}{c}47.07 \\
(10.78)\end{array}$ & $\begin{array}{c}48.41 \\
(11.00)\end{array}$ & $\begin{array}{l}46.30 \\
(8.85)\end{array}$ & $\begin{array}{c}46.67 \\
(11.10)\end{array}$ & 1.418 & 0.227 \\
\hline
\end{tabular}

Note: $\quad$ 1.The value within bracket refers to standard deviation. 


\section{FINDINGS}

- The findings reveal that Risk taking Propensity contributes significantly to the respondents' intention to become entrepreneurs based on their gender, course of study, birth region. It was found that under graduate students have more Risk taking Propensity than post graduate students. Risk taking Propensity contributes significantly to the respondents' intention to become entrepreneurs based on their work experience. Students with work experience have more Risk taking Propensity to become entrepreneurs than students without work experience. With regards to demographic variables and Risk taking propensity the results do not support any significant difference as regards to birth region, family type, students' birth order, level of study, occupation of mother and family income.

- With regards to demographic variables and Internal Locus of Control the results do not support any significant difference as regards to gender, course of study, birth region, family type, birth order and family income. There is significant difference between students with work experience and students without work experience with respect to Internal Locus of Control. There is significant difference between occupation of father with respect to Internal Locus of Control.

- Attitude towards Entrepreneurship contributes significantly to the respondents' intention to become entrepreneurs based on their gender, course of study, work experience and occupation of father. However, there is no significant difference between students place of residence, region of birth, type of family, students birth order, occupation of mother and monthly family income with respect to Attitude towards Entrepreneurship..Students from rural back ground have more Attitudes towards Entrepreneurship, than students from urban background. Students from North India have more Attitude towards Entrepreneurship than students from South India.

- There is significant difference between gender, place of residence, region of birth, course of study, work experience and occupation of father with respect to Entrepreneurial Intent. There is no significant difference between students' family type, birth order, occupation of mother and monthly family income $r$ with respect to Entrepreneurial Intent.

- As regards to perceived barriers, there is no significant difference between gender, level of study, region of birth, birth order ,place of residence ,family type students, occupation of mother and monthly family income with respect to perceived barriers. Students from rural back ground have more Perceived Barriers to become entrepreneurs than students from urban background. However, respondents with B.A, B.B.A, B.Com and B.SC are significantly different from one another with respect to Perceived Barriers. Respondents with BA as their course of study perceive more barriers followed by respondents' pursuing B.Sc, B.Com and B.B.A as their course of study.

- Finally, there is no significant difference between students' gender, level of study, region of birth, place of residence, type of family, birth order, occupation of mother and monthly family income with respect to Perceived Support. Undergraduate students have more Perceived Support to become entrepreneurs than post graduate students. Students from rural back ground have more Perceived Support to become entrepreneurs than students from urban background. Students from South India perceive more barriers to become entrepreneurs than students from North India. There is significant difference between students' work experience and occupation of father with respect to Perceived Support.

\section{CONCLUSION}

In today's world entrepreneurs tend to play a vital role. The economic development of a country largely depends on their contribution. Entrepreneurs help in economic development by creating employment and prosperity. It is important to foster the entrepreneurial spirit among students who have the potential to become entrepreneurs. According to the research findings there is a need for development of an understanding of the individual entrepreneurs' characteristics and contextual 
factors which affect entrepreneurial intent while designing policies for entrepreneurial development, especially among students. It is important for academicians, policy makers and government officials to understand the need to enhance the entrepreneurial intention level of the students. This may be done through skill development program, research conference on the entrepreneurial development, industrial training, and risk taking capacity development among various other such initiatives. The results of this study indicate that demographic profile of the students may play an important role in recognizing potential entrepreneurs in the campus. It will help in the designing of programmes and initiatives which will be instrumental in nurturing young minds to consider entrepreneurship as a career choice.

\section{REFERENCES}

[1] Austin J H, Stevenson and J.Wei Skillen (2006),Social and Commercial Entrepreneurship: Same, different or both, Entrepreneurship Theory and Practice 30(1) 1-22,

[2] Bonnett, C. \& Furnham, A. (1991). Who wants to be an entrepreneur? A study of adolescents interested in a young enterprise scheme. Journal of Economic Psychology, 12, 465-478.

[3] Boyd, N. G., and G. S. Vozikis. 1994. The influence of selfefficacy on the development of entrepreneurial intentions and actions. Entrepreneurship: Theory \& Practice 18 (4): 63-77.

[4] Bruni, A., Gherardi, S., Poggio, S. (2004). Entrepreneurmentality, gender and the study of women entrepreneurs. Journal of Organization Change Management, 17(3), 256268.

[5] Cantillion, R. (1775), Essai sur la nature du commerce en general. London and Paris: Fetcher Gyles; Edited and translated by Henry Higgs (1931), London: MacMillan

[6] Cromie, S. (2000), "Assessing entrepreneurial inclinations: some approaches empirical evidence", European Journal of Work and Organizational Psychology, Vol. 9 No. 1, pp. 7-30.
[7] Douglas, E and D Shephard (2002). Self-employment as a career choice: Attitudes, entrepreneurial intentions and utility maximization. Entrepreneurship Theory and Practice, 26(3), 81-90.

[8] Gasse, Y. (1985), "A strategy for the promotion and identification of potential entrepreneurs at the secondary school level", in Hornaday, J.A., Shils, B., Timmons, J.A. and Vesper, K.H.(Eds), Frontiers of Entrepreneurship, Babson College, Wellesley, MA, pp. 538-59.

[9] Hisrich, R. D. and Peters, M. P. (1995), Entrepreneurship. Starting, developing, and managing a new enterprise: Homewood.

[10] Kamhon Kan, and Wei-Der Tsai. 2006. "Entrepreneurship and Risk Aversion." Small Business Economics 26 (5) (June): 465.

[11] Knight, F.H. (1921) Risk, Uncertainty and Profit (New York: Harper).

[12] Kolvereid, L. (1996a), Organizational employment versus self employment: reasons for career choice intentions, Entrepreneurship Theory and Practice, 20(3), pp. 23-3l.

[13] Kolvereid, L. (1996b), 'Prediction of employment status choice intentions', Entrepreneurship Theory and Practice, 20(3), pp. 45-57.

[14] Krueger, N, M Reilly and Carsrud, A.L (2000). Competing models of entrepreneurial intentions. Journal of Business Venturing, 15, 411-432.

[15] Krueger, N. F. and Carsrud, A. L. (1993), 'Entrepreneurial intentions: applying the theory of planned behavior', Entrepreneurship and Regional Development, 5, pp. 315330.

[16] Luthje, C., \& Franke, N. 2003. The 'making' of an entrepreneur: testing a model of entrepreneurial intent among engineering students at MIT. R\&D Management, 33(2): 135-148.

[17] Rotter, J. B. (1966) "Generalized expectancies for internal versus external control of reinforcement". Psychological Monograph, 80 (1) 609.

[18] Say.J.B. (1821) A treatise on political economy. Sherwood, Neely and Jones, London.

[19] Shapero, A. and Sokol, L. (1982). "The social dimensions of entrepreneurship". In C. A. Kent, D. L. Sexton, \& K. H. Vesper (eds.), Enclopaedia of Entrepreneurship, pp. 72-79 Englewood Cliffs, NJ: Prentice Hall. 